\title{
Acoustic imaging and collimating by slabs of sonic crystals made from arrays of rigid cylinders in air
}

\author{
Liang-Shan Chen \\ Department of Physics, Fudan University, Shanghai, China \\ Chao-Hsien Kuo and Zhen Ye* \\ Wave Phenomena Laboratory, Department of Physics, \\ National Central University, Chungli, Taiwan, China
}

(Dated: June 28, 2018)

\begin{abstract}
We show some new properties of the acoustic propagation in two-dimensional sonic crystals, formed by parallel rigid cylinders placed in air. The transmission through slabs of sonic crystals and the associated band structures are considered. It is shown that within partial bandgaps, the waves tend to be collimated or guided into the direction in which the propagation is allowed. Such a feature also prevails in the situations in which deaf bands appear. We show that within the partial bandgaps, a stable imaging effect can be obtained for flat sonic crystal slabs, in analogy to the cases with photonic crystals.
\end{abstract}

PACS numbers: $43.20 .+\mathrm{g}, 43.90 .+\mathrm{v}$

*Electronic address: zhen@phy.ncu.edu.tw 
The exciting phenomenon of band structures in sonic crystals allows for many possible applications, such as sound shields and acoustic filters [1, 2, 3, 4]. These applications mostly rely on the existence of complete sonic bandgaps in which acoustic waves are prohibited from transmission in all directions. Later, it was experimentally demonstrated [5] that proper SCs can also make refractive devices such as Fabry-Perot interferometers and acoustically converging lenses. The mechanism for the acoustic refractive devices lies in the linearly dispersive response of SCs in the low frequency regimes. In this case, it has been shown that lenticular SCs not only can behave as normal acoustic lenses, but also the focusing effect can be well described by Lensmaker's formula 6 ] .

Recently, a new property of photonic crystals has been discovered and has attracted much attention[7]. That is, it was suggested[8] that in certain frequency regimes, a flat slab of PCs not only is able to make focused images, but also such an imaging may be related to the superlensing phenomenon, a concept conceived from the study of the so called left-handed materials [9]. Further explorations [10, 11] indicate that good quality imaging can be indeed formed across flat photonic crystal slabs, and the overall imaging properties are mainly governed by the wave-guiding or self-collimation effects, in the presence of partial bandgaps or anisotropic wave scattering. The flat PCs slab imaging has subsequently been observed in the experiment [12, 13]. These discoveries are expected to pave a new avenue for photonic crystal applications in controlling optical flows 14].

Due to the similarities between acoustic and EM waves, acoustic imaging by flat slabs of sonic crystals may be also possible. In the present paper, we wish to explore this possibility, following the work done on photonic crystals $[8,10,11]$. We will study the imaging and wave-guiding effects of flat sonic crystal slabs and find the underlying mechanism for the imaging. The results reported here may lead to new applications of sonic crystals, such as medical imaging, and manipulation of acoustic flows.

The sonic crystal systems are from the existing experiments [5]; in this way, we expect that experimental verifications may be readily performed. Consider $N$ straight rigid cylinders located in air. An acoustic line source transmitting monochromatic waves is placed at a certain spatial point. The scattered wave from each cylinder is a linear response to the incident waves which are composed of the direct wave from the source and the multiply scattered waves from other cylinders, and subsequently contributes to the total waves. Such a scattering process has been first formulated exactly by Twersky [15]. In this paper, we will 
use Twersky's formulation to calculate the multiple scattering in the systems. For regular arrays of cylinders, the band structures are computed by the plane-wave method.

In the simulation, the rigid cylinders of radius $a$ are arranged to form square arrays in air with lattice constant $d$. The following parameters from the experiment [5] are used in the simulations: (1) the radius of the cylindrical rods is $1.5 \mathrm{~cm}$; (2) the lattice constant is $11.0 \mathrm{~cm}$; (3) The filling factor, defined as the area occupation of the cylinders per unit area $f_{s}=\pi(a / d)^{2}$, is equal to $0.05842 ;$ (4) the sound speed in air is $v_{a}=334 \mathrm{~m} / \mathrm{s}$. Additionally, the acoustic transmission through the cylinder arrays is normalized as $T=p / p_{0}$. The acoustic intensity field is defined as $|P|^{2}$. We note here that the rigid cylinders can be any solid rods. As shown in Ref. [2], any material whose acoustic impedance with respect to the air exceeds roughly 10 can be used as the composition material for the rods.

Figure 1 presents the band structure and transmission result for the square array of rigid cylinders. In the figure, the curves on the left denote various dispersion bands when the wave is propagating in different directions. The inserted box denotes the first Brillouin zone. The results in Fig. [1 reproduce nicely that in the experiment [5]. Here is shown that there is a partial bandgap ranging from 1.42 to $1.64 \mathrm{KHz}$, while a deaf band region appears from 2.08 to $2.72 \mathrm{KHz}$. The partial gap and the deaf band regimes are denoted by the horizontal lines respectively. Within the partial gap, the waves are prohibited from propagation along the $\Gamma X$ direction, i. e. the [10] direction. For frequencies inside the deaf band, the propagation is inhibited along the $\Gamma M$ direction, i. e. the [11] direction.

We find that the flat slab imaging can be formed for frequencies located within these regimes, in analogy with the photonic crystal cases. For brevity, we choose two frequencies for simulation, that is $1.50 \mathrm{KHz}$ and $2.11 \mathrm{KHz}$ which are within the partial gap and the deaf band respectively. Hereafter we denote the two cases as Case $\mathrm{G}$ and Case $\mathrm{C}$ respectively.

Figure 2 shows the imaging fields $|P|^{2}$ for two slabs of sonic crystals at frequencies 1.5 $\mathrm{KHz}$ and $2.11 \mathrm{KHz}$, i. e. Case G and Case D, respectively. For Case G in (a1) and (a2), the array measures as $7 \sqrt{2} d \times 36 \sqrt{2} d$, while the slab has the size of $9 d \times 49 d$ for Case D in (b1) and (b2). The orientations of the slabs are denoted in (a1) and (b1): the transmission is along [11] in Case G and along [10] in Case D. The acoustic source is placed at one lattice constant away from the left sides of the slabs. The results for Case $\mathrm{G}$ and Case D are similar. Here, we observe separately two well focused image points on the right hand sides of the two slabs. When looking at the intensity fields inside the two slabs ((a2) and (b2)), we find that 
the transmission is mainly confined within a pipeline or tunnel along $\Gamma M$ for Case $\mathrm{G}$ and along $\Gamma X$ in Case $\mathrm{D}$, and the waves travel to the other side of the slab, then release into the free space. This type of self-collimating or guiding behavior has been previously discussed for photonic crystals [10, 11].

The imaging and wave-guiding results in Fig. 2 may be understood in the framework of the band structure results. The band structure from Fig. 1 clearly shows that in the frequency ranges considered, there are a band gap along the $\Gamma X$ direction, and deaf band region along the $\Gamma M$ directly. Therefore the waves are prohibited from propagation along these two directions for Case $\mathrm{G}$ and Case D respectively. In other directions such as $\Gamma M$ for Case $\mathrm{G}$, however, there is an allowed band to support the wave propagation. To be specific, we consider Case $\mathrm{G}$ as the example. In the present setup, the incident wave is set along the $\Gamma M$ direction, which makes an angle of 45 degrees to the $\Gamma X$ direction. Since being prohibited from propagating in the $\Gamma X$ direction, i. e. 45 degrees from the straight horizontal direction, waves tend to move forward along the allowable $\Gamma M$ direction. The frequency band in the $\Gamma M$ direction thus provides a propagating avenue for the waves to go over to the other side of the slab, forming the images. As to be published elsewhere, we note that the results from Fig. 2 may not be explained by the equal-frequency method proposed in Ref. [8].

To further support our observation, we place a transmitting source inside two arrays of cylinders. The overall shape of the array is square. Fig. 3 presents the simulation results. The cylinders are arranged to form a square arrays whose sides measure respectively $14 \sqrt{2} d$ for Case G and 19d for Case D. Same as in Fig. 2, the orientations of the slabs are denoted in (a1) and (b1). The intensities for waves outside or inside the slabs are shown in the (a1), (b1) and (a2), (b2) for the two cases. As expected, the focused images are evident in four allowed directions for each case: in the $[1,1],[-1,1],[-1,-1]$, and $[1,-1]$ directions for Case $\mathrm{G}$, and in the $[1,0],[0,1],[-1,0]$, and $[0,-1]$ for Case D. The field images inside the arrays clearly show the travelling path of the waves along these directions, depicted by (a2) and (b2).

The above flat slab imaging and guiding results are qualitatively similar for both partial gaps and the deaf bands. Further simulations indicate that the imaging effect for partial gaps is insensitive to either source location nor the slab horizontal size, revealing a stable imaging. For the deaf band situation, however, the cross slab imaging can depend on the the location or the slab size. In Figs. 4 and 5 we show the imaging effects for two variations 
of the results in Fig. 2, In Fig. 4, the slab sizes have been increases to be $11 \sqrt{2} d \times 36 \sqrt{2} d$ for Case G and $15 d \times 49 d$ for Case D. In Fig. 5, the source is moved one more lattice constant away from the slab. Here, we observe that the imaging patterns for Case G do not change: there is still a single well formed imaging point with nearly the same distance to the slab. In Case $\mathrm{G}$, however, the single imaging point is split into two focused points. The feature of guided travelling paths inside the slabs, however, remains unchanged for both cases.

In summary, following the recent development in the photonic crystal research, we propose some new properties of sonic crystals. That is, a flat slab of sonic crystals can make well focused images. This peculiar effect can be caused by either the partial bandgaps or the deaf bands. It is also found that the partial bandgaps or the deaf bands can guide wave propagation into passing band directions.

The work received support from National Science Council. We greatly appreciate the correspondence with M. Nieto-Vesperinas, J. Sanchez-Dehesa, J. Zi, and J. Pendry.

[1] R. Martínez-Sala, J. Sancho, J. V. Sánchez-Pérez, J. Llinares, and F. Meseguer, Nature (London) 378, 241 (1995).

[2] J. V. Sánchez-Pérez et al., Phys. Rev. Lett. 80, 5325 (1998).

[3] W. M. Robertson and W. F. Rudy III, J. Acoust. Soc. Am., 69, 3080 (1992).

[4] M. S. Kuswaha, Appl. Phys. Lett., 70, 3218 (1997).

[5] F. Cervera, et al., Phys. Rev. Lett., 88, 023902 (2002).

[6] C.-H. Kuo and Z. Ye, cond-mat/0312289.

[7] Refer to the survey in L. Venema, Nature, 420, 119 (2002).

[8] C. Luo, S. G. Johnson, J. D. Joannopoulos, and J. B. Pendry, Phys. Rev. B 65, 201104(R) (2002); M. Notomi, Phys. Rev. B 62, 10696 (2000).

[9] J. B. Pendry, Phys. Rev. Lett. 85, 3966 (2000).

[10] Z.-Y. Li and L.-L. Lin, Phys. Rev. B 68, 245110 (2003).

[11] C.-H. Kuo and Z. Ye, cond-mat/0310423.

[12] P. V. Parimi, W.-T. Lu, P. Vodo, and S. Sridhar, Nature 426, 404 (2003).

[13] We note that the interpretation of the slab imaging effects in 12] is based upon the theoretical analysis in Ref. [8]. The latter has been questioned in Refs. [10, 11]. 
[14] e. g. L.-S. Chen, C.-H. Kuo, and Z. Ye, physics/0312126.

[15] V. Twersky, J. Acoust. Soc. Am. 24, 42 (1951). 


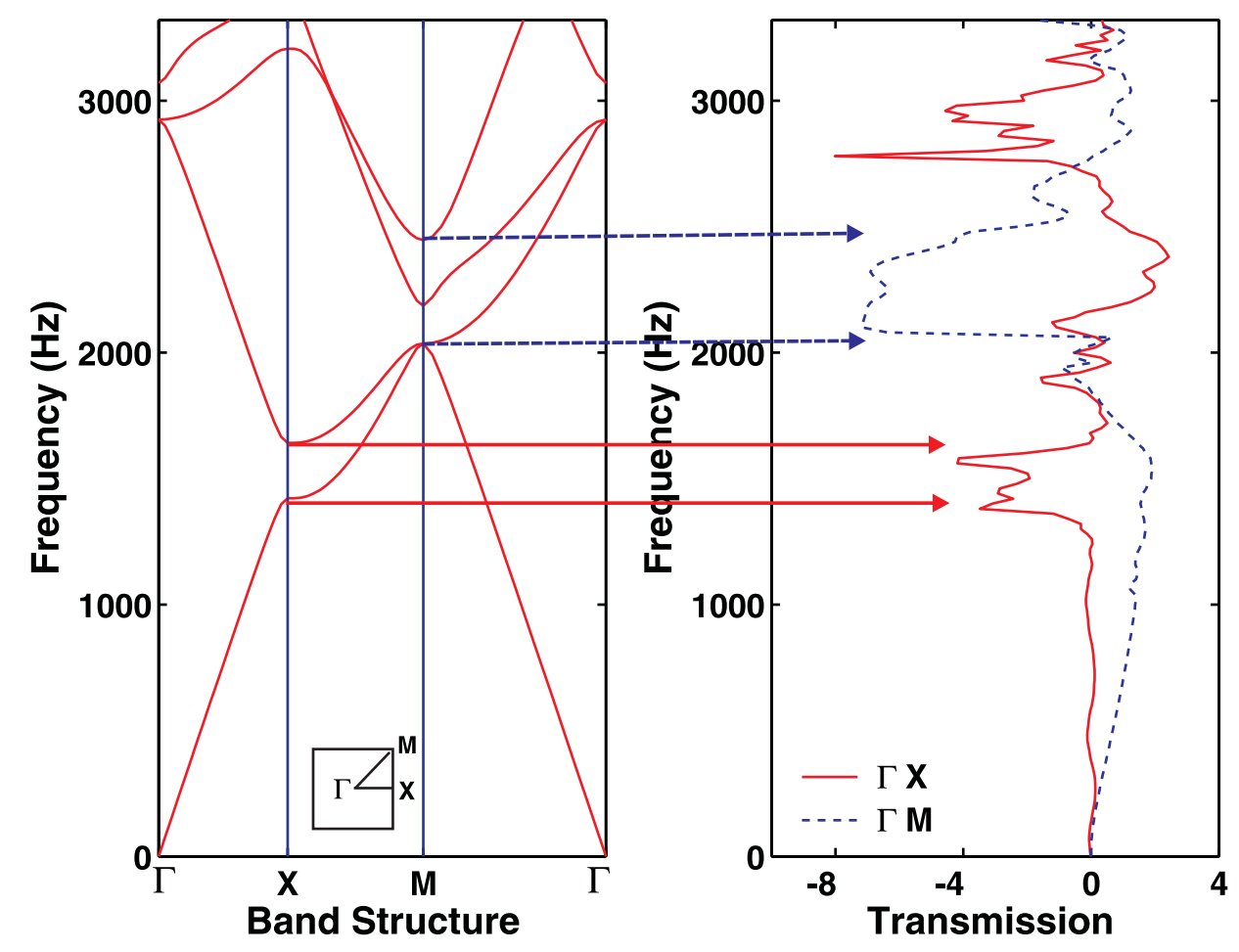

FIG. 1: Left panel: the band structure of a square lattice of rigid cylinders in air. A partial gap is between the two horizontal lines. Right panel: the normalized transmission versus frequency. 

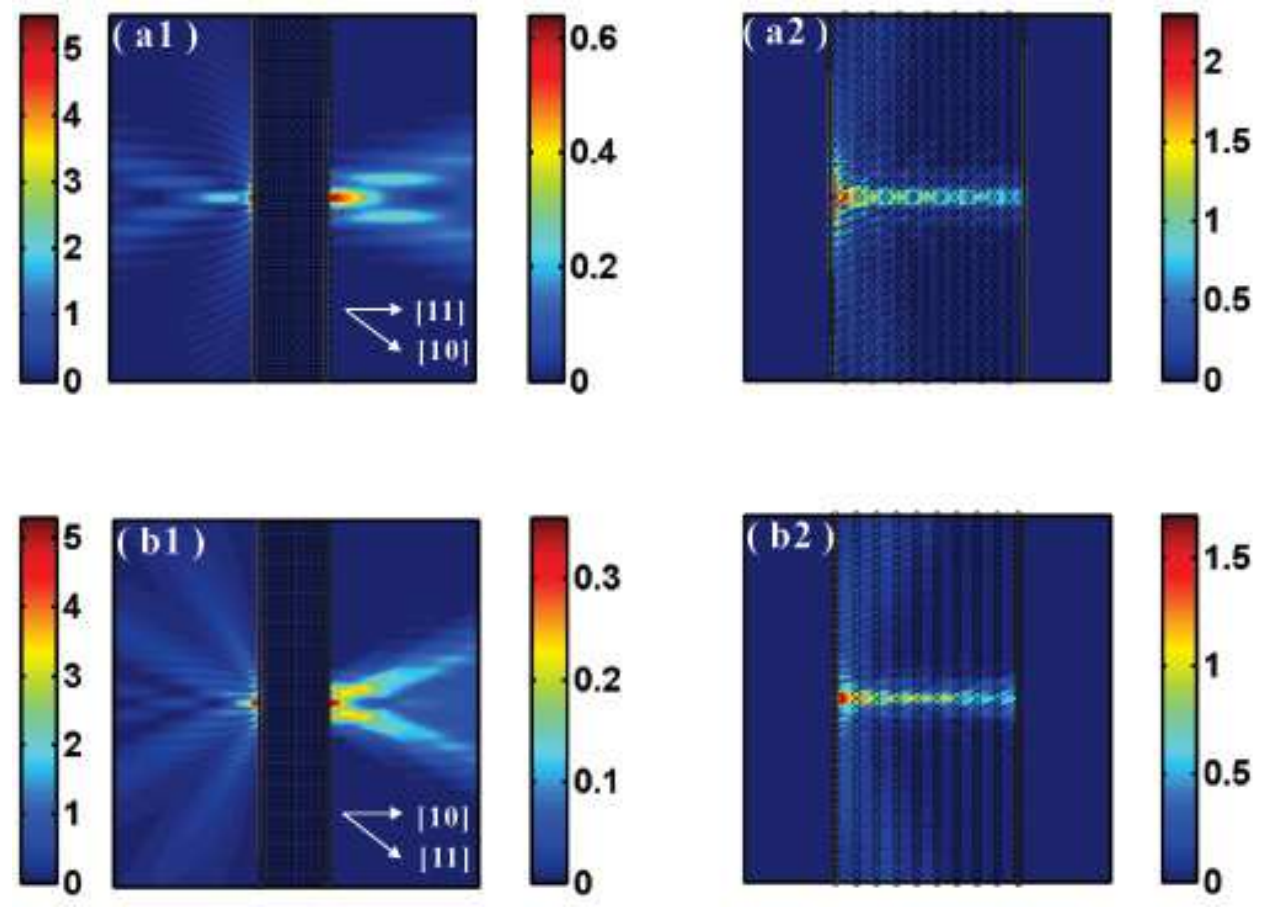

FIG. 2: The imaging fields for two slabs of sonic crystal structure for two frequencies $1.50 \mathrm{KHz}$ and $2.11 \mathrm{KHz}$, located within the partial gap and the deaf band area respectively. (a1) and (b1) show the intensity field outside the slab. (a2) and (b2) plot the intensity fields for the areas inside. Here we see clearly that the waves propagate mainly in a small tunnel inside the slab for both frequencies, and make the focused images across the slab. 

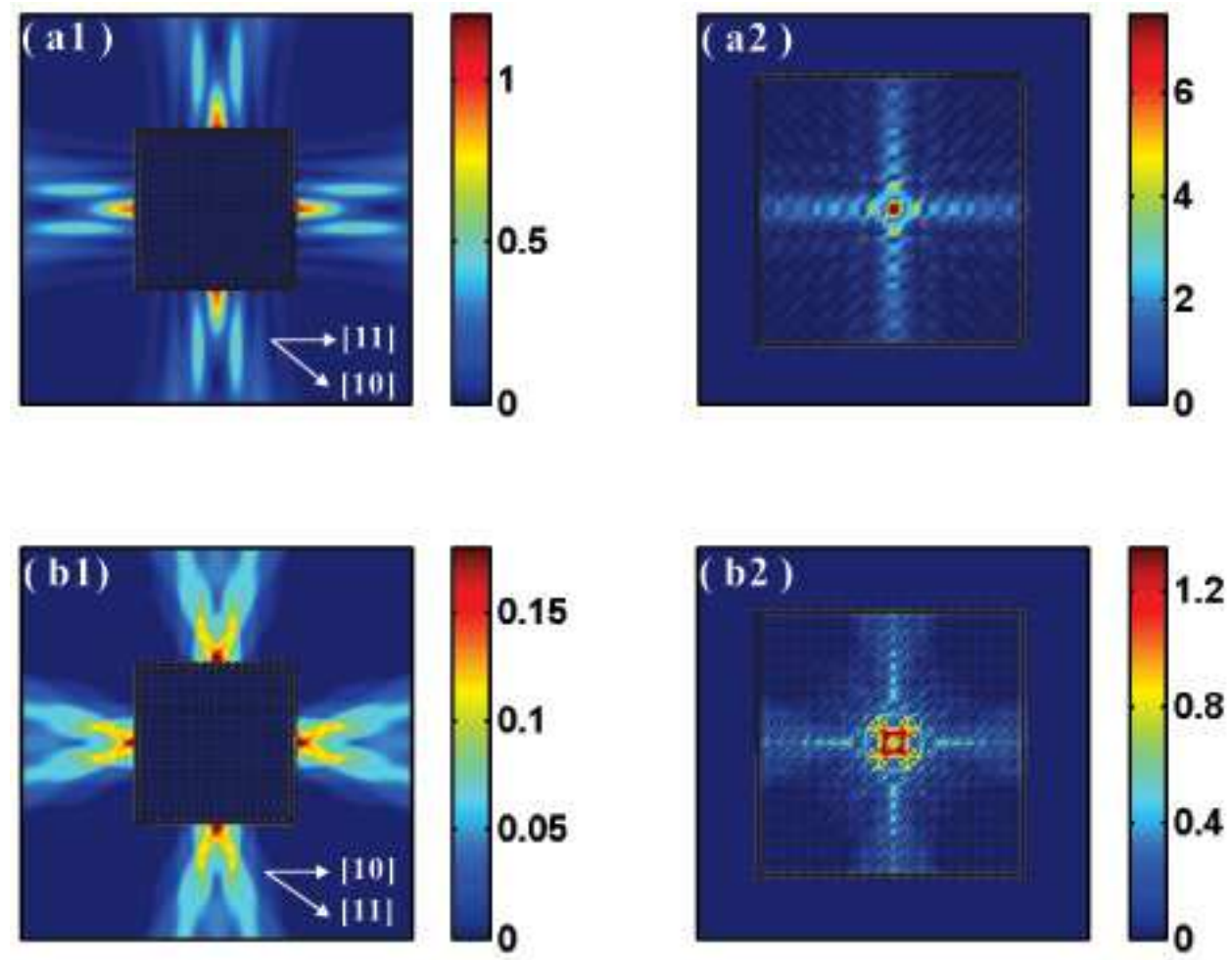

FIG. 3: The imaging fields for a transmitting source located inside two square arrays of cylinders. In (a1) and (a2), the square measures $14 \sqrt{2} \times 14 \sqrt{2}$, while the square array has the size of $19 \times 19$ in (b1) and (b2). All other parameters are the same as in Fig. 2. (a1) and (b1) show the intensity field outside the slab. (a2) and (b2) plot the intensity fields for the areas inside the slabs. 

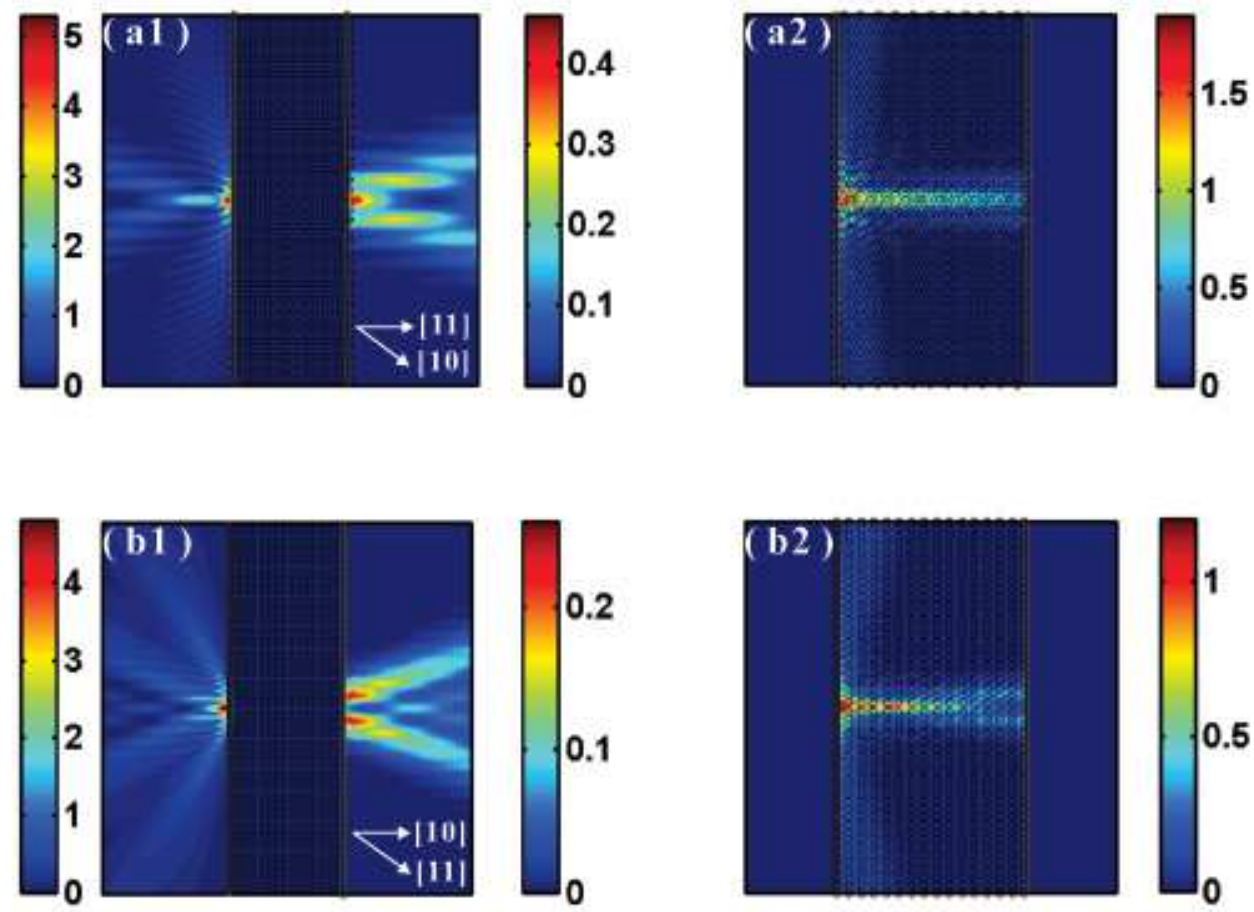

FIG. 4: The imaging fields for two slabs of sonic crystal structure, similar to Fig. 2 but with larger sample lengths. 

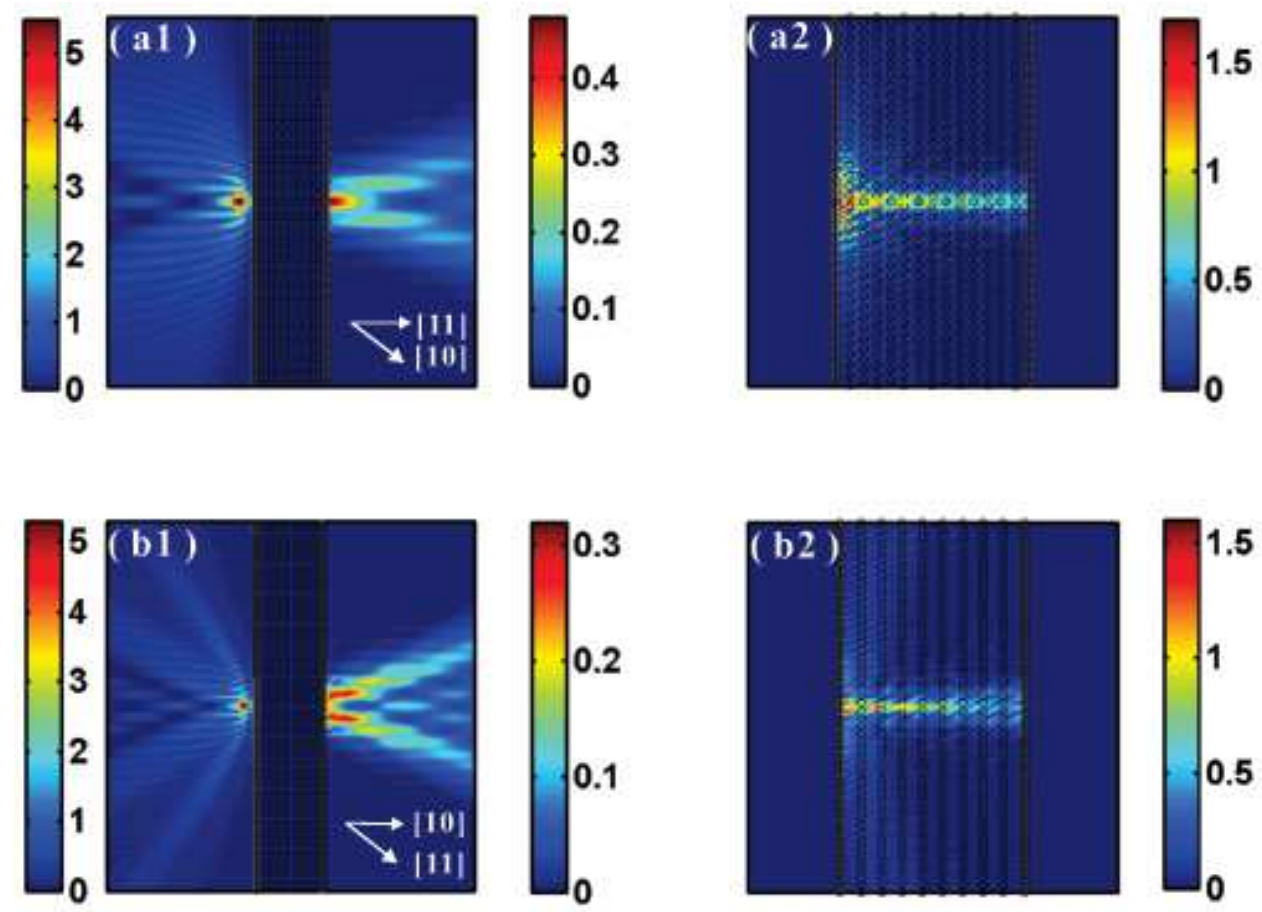

FIG. 5: The imaging fields for two slabs of sonic crystal structure, similar to Fig. 2 except that the source has been moved one more lattice constant away from the slabs. 